\title{
A Conversation Between Hedvig Hricak and Johannes Czernin
}

\author{
Hedvig Hricak $^{1}$ and Johannes Czernin ${ }^{2}$ \\ ${ }^{I}$ Department of Radiology, Memorial Sloan Kettering Cancer Center, New York, New York; and ${ }^{2}$ David Geffen School of Medicine at \\ UCLA, Los Angeles, California
}

F Czernin talked with Hedvig Hricak, MD, PhD, chair of the Department of Radiology at Memorial Sloan Kettering (MSK) Cancer Center (New York, NY). She holds a senior position within the Program of Molecular and Pharmacology Therapeutics at the Sloan Kettering Institute and is a professor of Radiology at the Weill Medical College of Cornell University (New York, NY). She received her medical degree from the University of Zagreb (Croatia) in 1970. After beginning her residency at Hospital M. Stojanović in Zagreb, she continued residency at St. Joseph Mercy Hospital (Pontiac, MI) from 1974 to 1977. In 1978, she completed a fellowship in radiology, concentrating on CT and ultrasound, at Henry Ford Hospital (Detroit, MI), where she stayed on as a faculty member from 1979 to 1981 . She also served as an assistant clinical professor of diagnostic radiology at the University of Michigan (Ann Arbor). In 1982, she moved to the University of California, San Francisco (UCSF), and, in 1986, became a professor of radiology with joint appointments in urology; radiation oncology; and obstetrics, gynecology, and reproductive sciences. She earned her DrMedSc (PhD equivalent) degree from the Karolinska Institute (Stockholm, Sweden) in 1992. In November 1999, she assumed her current role as chair of radiology at MSK.

Dr. Hricak has participated in the development of numerous technologic innovations in ultrasound, MR, and CT imaging for gynecologic cancers and in MR and MR spectroscopy for prostate cancer. Her research focuses on the evidence-based use of imaging in cancer management, including detection, treatment selection, treatment planning, and treatment follow-up. She is the current president of the Academy for Radiology and Biomedical Imaging Research and a past president of the Radiologic Society of North America (RSNA), the International Society for Strategic Studies in Radiology, the Society for Advancement of Women's Imaging, the California Academy of Medicine, the Society of Uroradiology, and the New York Roentgen Society. She is a member of the National Academy of Medicine (NAM) and a fellow of the American College of Radiology, the International Society for MR in Medicine, and the Society of Uroradiology. Dr. Hricak has authored or coauthored almost 600 peer-reviewed articles and numerous book chapters and other publications. In recognition of her career accomplishments, she has received the gold medals of the Radiologic Society of North America, the International Society for MR in Medicine, the Asian Oceanian Society of Radiology, and the European Society of Radiology; the Béclère medal of the International Society of Radiology; the Schinz Medal of the Swiss Society of Radiology; the David Rall Medal of the NAM; the

COPYRIGHT @ 2019 by the Society of Nuclear Medicine and Molecular Imaging.
Howard Pollack Medal of the Society for Abdominal Radiology, the Morocco Medal of Merit, the Jean A. Vezina French Canadian Award of Innovation, and the Order of the Croatian Morning Star Katarina Zrinska Presidential Award of Croatia.

Dr. Czernin: I want to start out with a few questions about your career. You

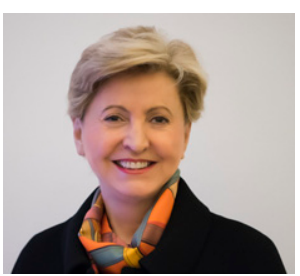

Hedvig Hricak, MD, PhD were trained at the University of Zagreb in Croatia. Why did you move to the United States?

Dr. Hricak: Thank you for inviting me. I finished my medical school in Zagreb, where I did my internship and took the mandatory state examination that allowed me to practice general medicine and continue with my residency. I started my residency in radiology. As you know, radiology is a very technology-dependent subspecialty, and I quickly recognized that opportunities for advanced clinical practice and research were limited. I wanted to explore the opportunities and expand my horizons. After passing my qualifying examinations for the United States, I went to Detroit, finished my residency in a small community hospital, and continued with my fellowship in ultrasound and CT at Henry Ford Hospital. This is how my career started. I was fortunate that William R. Eyler, MD, at that time was the chair of radiology at Henry Ford. Dr. Eyler was a superb radiologist, dedicated mentor to his faculty, excellent teacher, and fierce advocate for research. He was also the editor of Radiology. He embraced my ambitions. He took me under his wing. He was fair and tough. He asked me to revise my first paper 21 times-and this was before computers! Each time I had to retype the entire manuscript. It was not easy, but I learned a lot. He also encouraged me to apply for a grant and explore preclinical research. I received funds from the Henry Ford Foundation and started my research program in ultrasound of kidney transplants in canines. This was at a time when both ultrasound technology and renal transplantation were just starting to be widely disseminated. The research project was innovative and exciting. Sometimes, you are just lucky to be at the right place at the right time, and I consider myself to have been very fortunate.

In January 1982 I went to the UCSF. I arrived at a time when there were only 2 clinical MR machines in the United States, and one of them was at UCSF. Clinical MRI was in its infancy. The excitement as we were seeing in vivo details of human anatomy was beyond words. This constellation of "lucky stars" launched my research career. I received my first National Institutes of Health (NIH) R01 grant in 1989. Not only do I find clinical research exciting, but I also believe that by advancing the field and exploring new frontiers, you are always pushing the clinical envelope and discovering new findings or methodologies that improve patient care. I 
love clinical radiology. You combine clinical and imaging findings, you solve many puzzles, and you continuously learn.

I always liked challenges, and leadership is one of them. Going through my career I appreciated the opportunities to lead my lab, a genitourinary section, an abdominal imaging section, and now, of course, to be a department chair.

Dr. Czernin: When you became the chair of radiology at MSK in the late 1990s, no women were in leadership positions. True?

Dr. Hricak: I officially started my MSK chairmanship on November 1, 1999. At that time, I was the only female chair of radiology in the United States.

Dr. Czernin: How has that changed at MSK?

Dr. Hricak: Today we have 5 women department chairs at MSK, and there are many more female chairs of radiology in the United States.

Dr. Czernin: What was the status of the department at that time, and what were the initial changes you made?

Dr. Hricak: From the day of my first interview at MSK, I liked the environment and the people very much. I like being in a focused environment, and at MSK we all are dedicated to one goal: improving cancer care. In 1999, the department was small, with only 29 faculty members. I was able to get to know each faculty member, while participating in a clinical rotation and doing my own research. Today we have 171 faculty members and more than 1,500 staff, and we practice in 9 geographically dispersed locations. My role has evolved and changed tremendously. My role today is taking care of departmental needs-building the infrastructure necessary for started a new educational track partnering with radiation oncology.

Dr. Czernin: I read one of your fairly recent papers in Radiology, where you provide your vision of the key focal areas for the future. I'm going to list a few here. One is, of course, big data and how big data can be managed; artificial intelligence (AI) was another. In the context of technology advances you mentioned image-guided therapy, both interventional and noninterventional, and targeted radionuclide therapy. You also talked about radiomics and radiogenomics. Are your priorities at MSK more instrument driven, or are they more focused on developing personalized oncology? Or are they driven by extracting as much data from these huge datasets as possible and merging the genomic data with imaging data plus proteomics data and so on?

Dr. Hricak: All of the above. The reason for embracing technologic advances is to build an infrastructure that allows the faculty to excel. One of the first technology-driven initiatives I worked on at MSK was to build an image-guided oncologic interventional program, today known as the Center for Image-Guided Intervention. I believe it is still among the most modern interventional radiology (IR) oncology centers in the world. We were early proponents of CT angiography, which we believed was going to be essential for the future of IR. In the mid-2000s, the enthusiasm for CT angiography as opposed to angiography alone was not widely shared. We also introduced PET/CT as essential equipment in an interventional suite. Molecular medicine was exploding, and it was obvious that in no time there would be a need to perform biology-driven and not

\section{"Al applications, once they are more fully developed, will help our specialties to streamline, enhance, accelerate, personalize, and standardize every aspect of our daily work. They will improve the patient experience, eliminate repetitive tasks that today are mostly done manually, and help us more consistently improve the quality of our reports and the quality of our own work life."}

delivering outstanding care and allowing our faculty to excel in clinical work, research, and education. Not only our clinical volume but also our research programs have expanded greatly, and I am proud that our clinical and research faculty are among the best in the world.

Dr. Czernin: You became the president of the RSNA in 2010. I'm sure that you had some programmatic goals at that time. What kind of forward-looking changes did you want to implement in the society as a vision for the entire field of radiology?

Dr. Hricak: Being able to serve on the RSNA Board of Directors is an honor, and it is a great opportunity to contribute to your specialty both in education and research, influencing the road for the future. To do that you need not only vision but also passion and persuasiveness, as every decision of the RSNA board is consensus driven. Board appointments are for $8 \mathrm{y}$, and throughout my service I learned. At the time of my appointment to the board, molecular imaging was in its infancy. The board agreed that molecular imaging held tremendous potential for our future, and we started small, with a committee and a few lectures. The rest is history. Not surprisingly, my second greatest passion was improving education in oncologic imaging, and the third was promoting an interdisciplinary approach, understanding that we radiologists are consultants and not just film readers. We just anatomically localized biopsies or tumor ablations. Having a superb IR infrastructure gives our IR faculty an unprecedented opportunity to practice at the cutting edge and to advance their research careers and grant portfolios. Through their work, such as with molecularly driven biopsies and the development of targeted therapies, including theranostics, they are also helping other medical specialties to advance. At present, our priorities include further developing targeted molecular imaging and developing and implementing theranostics. To realize those opportunities, we are expanding our radiochemistry facilities and building an $\alpha-\mathrm{lab}$, adding to our existing cyclotron facility and state-of-the-art Good Manufacturing Practice radiopharmacy lab. We are also expanding and reorganizing our clinic space dedicated to theranostics. Bricks are important, but they serve as an infrastructure. Programs are built by people, and, for example, in nuclear medicine, we are truly fortunate to have been able to recruit stars like Jason Lewis, PhD, Heiko Schöder, MD, MBA, and Lisa Bodei, $\mathrm{MD}, \mathrm{PhD}$. And it certainly helps that one of the fathers of modern nuclear medicine, Steven Larson, MD, is a loyal member of our department.

Dr. Czernin: Of your close to 600 articles, nearly 400 are on prostate cancer. What does your clinical prostate cancer imaging program look like? Do you use PET/MR? 
Dr. Hricak: I believe that in oncology, PET/MRI is still mostly in an exploratory phase. We are still searching for an innovative clinical application of PET/MRI that will provide true incremental value-meaning additional data that affects patient management. At present, we use PET/MRI in pediatric oncology because our pediatric patients often have long-term follow-up studies, and the radiation dose does make a difference. We also use PET/MRI in patients who need both PET/CT and MRI tests for their diagnostic work-ups - an application we refer to as a patient-friendly and patient-centric approach. In imaging prostate cancer, we use PET/MRI for pretreatment evaluation of aggressive disease and sometimes for evaluation of metastatic disease. In both indications, the value added consists mainly of greater convenience and comfort for the patient.

We are developing research projects exploring the use of PET/ MRI and new molecular probes. In addition, we are contemplating a study combining PET/MRI and hyperpolarized MR, because the dynamic information on pyruvate metabolism may give us new insights into disease biology.

Dr. Czernin: So you would say that the only very modest success of PET/MR is most likely explained by the lack of demonstration of incremental value thus far?

Dr. Hricak: Yes. I would love to hear your opinion.

Dr. Czernin: It was my opinion from the beginning that it's problematic because its purchase price and operational costs are much too high. And the idea that it will outcompete PET/CT was kind of naive. I think that MRI, if used to the best of its ability, is best done as a stand-alone procedure. If you just use it as a kind of replacement for $C T$, then it defeats the purpose.

Dr. Hricak: I am very glad that we agree. As you know, we may be in the minority. For me, facts speak volumes. For example, at MSK we have 15 PET/CTs and 1 PET/MRI. Sometimes we are asked why we don't have more PET/MRI scanners. We will acquire more as we observe the needs evolving.

Dr. Czernin: Some fear has permeated parts of radiology for a time that are related to AI. I think that this shouldn't make us fearful at all but actually help us relax a little bit, as there may be great progress in efficiency on the horizon. More important, integrating big imaging data with biology and pathology data could really improve patient care.

Dr. Hricak: I absolutely agree with you. AI applications, once they are more fully developed, will help our specialties to streamline, enhance, accelerate, personalize, and standardize every aspect of our daily work. They will improve the patient experience, eliminate repetitive tasks that today are mostly done manually, and help us more consistently improve the quality of our reports and the quality of our own work life. AI may even decrease burnout. I would like to give you a few examples of low-hanging fruits for AI, starting with automatic study protocols, personalized to each patient based on clinical indication, risk factors, and body type. MRI, CT, and PET/CT protocols currently consume a lot of physician time in oncology. For radiologists or nuclear medicine physicians, the process of protocoling and interpreting these examinations and searching through medical records for key information is quite labor intensive. We all know how inaccurate or incomplete the indication can be on the request. Optimal AI image acquisition will improve and standardize images. In postprocessing, automated extraction of information from abnormalities to allow better assessment of disease, automated SUV and tumor volume measurements, and graphic longitudinal display of tumor volume changes would be dreams come true for the oncologic radiologist or nuclear medicine physician. At the time of study review and interpretation, triage by AI, indicating a normal study or highlighting any study that requires more careful review, with a display of key images with quantitative actionable information, seems almost too much to dream of, but it may well be possible. I do not mean to imply that normal studies will not be looked at - they must be verified; however, the time required for verification will be much shorter. And then, ultimately, we can even hope for multimodal image analysis, because many of our patients undergo a combination of imaging studies, and manually performed comparison is very labor intensive. Those are just a few items on our wish list, all of which will not replace but rather enhance our profession.

Dr. Czernin: This brings me to the political part of our discussion. I have been promoting independent nuclear medicine departments for quite some time. Most of the great successes over the last 10 years in nuclear medicine and molecular imaging came from Europe and Australia, where nuclear medicine is an independent, well-integrated, but autonomous department in terms of administration and leadership. When you have well-informed chairpersons with an understanding of molecular imaging and theranostics there will be lots of support for nuclear medicine. This is, however, not the case in many radiology departments. So let me ask bluntly: Is there any reason for you to believe that nuclear medicine needs to be a division of radiology?

Dr. Hricak: It's a very good question, and I understand your point of view. However, as you know, even in Europe the practice is changing, and there are more and more universities where the two departments are joined. I think it's all about leadership. You need a chair who has vision, perseverance, courage, and understanding of the current and future landscape of diagnostic imaging, interventional therapy, and nuclear medicine. Sometimes as a chair, with such an increase in administrative duties and pressures on economic issues, it is so easy to forget the difference between managing and leading. Putting out daily fires within your department can be overwhelming, and leading for tomorrow can take a back seat. Commitment to departmental vision should never be put on hold. But there are broader, external fires that must be addressed as well. A recent example is the Nuclear Regulatory Commission's attempt to soften requirements for Authorized Users. As you know, the proposal was to adjust the training and experience requirements so that almost anyone could administer theranostics. As a chair of a radiology department, I joined other leaders from radiology and nuclear medicine, and we sent a strong letter opposing the change. The main issue is patient safety and the quality of studies. Authorized Users must be properly trained and understand radionuclides beyond FDG.

I don't know why nuclear medicine is relatively weak in this country, especially as compared with Europe or Australia. I would be delighted to join any efforts trying to solve the problem. Unless we are able to greatly enhance the appeal of training in nuclear medicine and recruit a large number of the brightest and best physicians to become nuclear medicine clinician/ scientists, regardless of which background they come from, we are jeopardizing the future of molecular imaging and theranostics, which I believe is one of the most promising areas in our field.

Dr. Czernin: My point is if people are not empowered and nuclear medicine is an appendage of radiology, as it is in most radiology 
departments, the chances of success are very low. So you ask the question of why nuclear medicine is weak in the United States. When specialties are not independent, the incentive to perform at a high level is not there. I agree with you: if you have a wise and competent leader, then divisions are fine. However, leadership with nuclear medicine and molecular imaging knowledge is available in only a very few large academic departments. Interest is limited, because nuclear medicine interferes with regular workflows. The same is true for radiation oncology departments: their workflow is not well suited for theranostics. Theranostics take too long. So I agree with you. We have the best example for precision medicine and precision oncology, but nuclear medicine needs to wake up and become independent and strong.
Could you give a 1- or 2-paragraph summary of what you see for the future for radiology and nuclear medicine?

Dr. Hricak: I am a big optimist. There are new horizons for diagnostic radiology, nuclear medicine, and IR. It depends on us, the steps that we take to embrace the opportunities that our fields offer for making discoveries, such as for advancing in vivo understanding of cancer biology and continuously improving cancer outcomes. This means incorporating not only advances in imaging but also imaging-based therapeutic approaches, such as image-guided interventions and theranostics, as essential components of cancer care.

Dr. Czernin: Thank you very much, Hedi. It was really interesting to talk to you, and I wish we could just continue. Thank you for your time. 